\title{
A Remark on the Global Lipschitz Regularity of Solutions to Inner Obstacle Problems Involving Degenerate Functionals of $p$-Growth
}

\author{
MARTIN FUCHS
}

\begin{abstract}
We extend some recent results of Jagodziński, Olek and Szczepaniak [Irish Math. Soc. Bull. 61 (2008), 1527 ] on the Lipschitz character of solutions to inner obstacle problems associated to a uniformly elliptic operator to the case of nonlinear, degenerate operators
\end{abstract}

In a recent paper Jagodziński, Olek and Szczepaniak [6] investigated the Lipschitz regularity of solutions to so-called inner obstacle problems extending earlier work of Jordanov [7]. By definition we are confronted with an inner obstacle problem if one or several side conditions imposed on the comparison functions are required to hold only on certain specified subregions of the domain of definition, where in case of several obstacles from above and/or below some natural conditions for compatibility have to be satisfied. The basic ideas for these kind of obstacle problems with obstacles defined only on a portion of $\Omega$ are explained in the textbook of Kinderlehrer and Stampacchia [8] (see pp. 137-139). The purpose of our short note now is the analysis of the inner obstacle problem for the $p$-energy functional

$$
I[u, \Omega]:=\frac{1}{p} \int_{\Omega}|\nabla u|^{p} d x
$$

with arbitrary exponent $p \in(1, \infty)$. Of course we could also consider the variational inequality associated to the operator

$$
L u:=-\sum_{i, j=1}^{n} \partial_{i}\left(A(x, \nabla u) a_{i j}(x) \partial_{j} u\right),
$$

2000 Mathematics Subject Classification. 49J40, 49N60, 35J85.

Key words and phrases. Inner obstacle problems, degenerate functionals, Lipschitz regularity of minimizers. 
where $a_{i j}(x)$ are smooth elliptic coefficients and where we have abbreviated $A(x, \nabla u):=\left(\sum_{\alpha, \beta=1}^{n} a_{\alpha \beta}(x) \partial_{\alpha} u \partial_{\beta} u\right)^{\frac{p}{2}-1}$, but this would not lead to a deeper insight. Note that in the above cited papers the case $p=2$ is considered. For simplicity we also just discuss the case of one inner obstacle (from below): in the presence of several inner constraints the arguments of [6] have to be modified in an obvious way, which in particular means that we have to impose the same natural assumptions of compatibility on the functions acting as side conditions and on their domains of definitions as done in [6].

Next we give a precise formulation of our hypotheses concerning the data: let $\Omega$ denote a bounded, open set in $\mathbb{R}^{n}$ whose boundary can locally be represented as a graph of a function with Hölder continuous derivatives. Suppose further that $\omega$ is an open subset of $\Omega$ with $\partial \omega$ being of the same regularity as $\partial \Omega$ and such that $\bar{\omega} \subset \Omega$. Let us consider a function $\Psi \in C^{1, \alpha_{1}}(\bar{\omega})$ for some $\alpha_{1} \in(0,1)$ and define the class of comparison functions

$$
\mathbb{K}:=\left\{w \in \stackrel{\circ}{W_{p}^{1}}(\Omega): w \geq \Psi \quad \text { a.e. on } \omega\right\},
$$

where $\stackrel{\circ}{W}_{p}^{1}(\Omega)$ is the usual Sobolev space of functions vanishing on $\partial \Omega$ as introduced for example in [1]. Then we have following result:

Theorem 1. The inner obstacle problem $I[\cdot, \Omega] \rightarrow \min$ in $\mathbb{K}$ with $I[\cdot, \Omega]$ and $\mathbb{K}$ being defined in (1) and (2) admits a unique solution $u \in \mathbb{K}$. The function $u$ is globally Lipschitz, moreover we have $u \in$ $C^{1, \alpha}\left(\bar{\Omega}-(\partial \omega)_{\varepsilon}\right)$ for some $\alpha \in(0,1),(\partial \omega)_{\varepsilon}$ denoting the set $\{x \in \Omega$ : $\operatorname{dist}(x, \partial \omega)<\varepsilon\}$.

Proof. Since $\mathbb{K} \neq \emptyset$, the existence and the uniqueness of a minimizer $u$ is immediate, and clearly $u$ is the solution of the variational inequality

$$
\int_{\Omega}|\nabla u|^{p-2} \nabla u \cdot \nabla(v-u) d x \geq 0 \quad \forall v \in \mathbb{K} .
$$

As done in [6] we will reduce (3) to a global obstacle problem with a suitable constraint $\widetilde{\Psi}: \Omega \rightarrow \mathbb{R}$. To this purpose consider the minimization problem

$$
I[w, \Omega-\bar{\omega}] \rightarrow \min , w \in \mathcal{C},
$$


where $\mathcal{C}:=\left\{w \in W_{p}^{1}(\Omega-\bar{\omega}):\left.w\right|_{\partial \Omega}=0\right.$ and $\left.\left.w\right|_{\partial \omega}=\Psi\right\}$, and let $h$ denote the unique solution of (4). We further define

$$
\widetilde{\Psi}:=\left\{\begin{array}{ll}
h & \text { on } \Omega-\omega, \\
\Psi & \text { on } \omega
\end{array}\right\} \in \stackrel{\circ}{W}_{p}^{1}(\Omega)
$$

and introduce the "global" class $\widetilde{\mathbb{K}}=\left\{w \in \stackrel{\circ}{W}_{p}^{1}(\Omega): w \geq \widetilde{\Psi}\right.$ a.e. on $\left.\Omega\right\}$ as well as the "global" problem

$$
I[\cdot, \Omega] \rightarrow \min \text { in } \widetilde{\mathbb{K}} .
$$

If $\widetilde{u} \in \mathbb{K}$ denotes the unique solution of (5), we claim the validity of

$$
u=\widetilde{u} .
$$

In fact, $\widetilde{u}$ is admissible in (3), i.e. we have

$$
\int_{\Omega}|\nabla u|^{p-2} \nabla u \cdot \nabla(\widetilde{u}-u) d x \geq 0 .
$$

(5) is equivalent to the variational inequality

$$
\int_{\Omega}|\nabla \widetilde{u}|^{p-2} \nabla \widetilde{u} \cdot \nabla(w-\widetilde{u}) d x \geq 0 \quad \forall w \in \widetilde{\mathbb{K}} .
$$

We like to insert $u$ into (8), which means that we have to check that

$$
u \geq h \quad \text { on } \Omega-\omega
$$

holds. From (3) it follows

$$
\int_{\Omega-\bar{\omega}}|\nabla u|^{p-2} \nabla u \cdot \nabla \varphi d x \geq 0 \quad \forall \varphi \in \stackrel{\circ}{W}_{p}^{1}(\Omega-\bar{\omega}), \varphi \geq 0,
$$

whereas we get from (4)

$$
\int_{\Omega-\bar{\omega}}|\nabla h|^{p-2} \nabla h \cdot \nabla \varphi d x=0 \quad \forall \varphi \in \stackrel{\circ}{W_{p}^{1}}(\Omega-\bar{\omega}) .
$$

The function $\varphi:=\max \{h-u, 0\}$ is in the space $\stackrel{\circ}{W}_{p}^{1}(\Omega-\bar{\omega})$ and for this choice of $\varphi$ (10) and (11) imply $\int_{M}\left[|\nabla u|^{p-2} \nabla u-|\nabla h|^{p-2} \nabla h\right]$. $\nabla(h-u) d x \geq 0, M:=(\Omega-\bar{\omega}) \cap[h>u]$, which by the coercivity of the field $\mathbb{R}^{n} \ni \xi \mapsto|\xi|^{p-2} \xi$ immediately gives $\nabla \varphi=0$ a.e. on $\Omega-\bar{\omega}$, i.e., $\varphi=0$ on this set, so that (9) follows. But then we combine (7) with (8) choosing $w=u$ and arrive at (6).

From the works of e.g. Evans [5], Di Benedetto [3], Lieberman [9], Manfredi [11, 12] and Tolksdorf [14] we deduce that the solution $h$ of 
problem (4) is of class $C^{1, \alpha}(\bar{\Omega}-\omega)$ for some $\alpha \in(0,1)$, in particular there is a finite constant $K$ such that

$$
|\nabla h| \leq K \quad \text { a.e. on } \Omega-\bar{\omega} .
$$

According to our assumptions $|\nabla \Psi|$ is in the space $L^{\infty}(\omega)$ so that on account of (12) $\widetilde{\Psi}$ is Lipschitz on the whole domain $\Omega$. (Note that we can not guarantee the validity of $\nabla h=\nabla \Psi$ on $\partial \omega$, which means that we do not know if $\widetilde{\Psi}$ is in $C^{1, \alpha}(\bar{\Omega})$.) This is enough to apply Theorem 1.2 of [2] with the result that the solution $\widetilde{u}$ of (5) and thereby $u$ (recall (6)) is locally Lipschitz in $\Omega$. The $C^{1, \alpha}$ - regularity of $u$ on the sets $\bar{\Omega}-(\partial \omega)_{\varepsilon}$ is consequence of the works of e.g. Choe and Lewis [4], Lieberman [10] or Mu and Ziemer [13]. This completes the proof of the Theorem, since obviously $|\nabla u| \in L_{\mathrm{loc}}^{\infty}(\Omega)$ together with $u \in C^{1, \alpha}\left(\bar{\Omega}-(\partial \omega)_{\varepsilon}\right)$ implies $|\nabla u| \in L^{\infty}(\Omega)$.

Remark 1. If we replace the energy from (1) by a more general functional $J[w, \Omega]:=\int_{\Omega} f(\nabla w) d x$, where $f$ should at least satisfy the hypotheses of Theorem 1.2 in [2], then again the global Lipschitz regularity of the minimizer will follow as soon as we can guarantee the global boundedness of $|\nabla h|, h$ being the solution of problem (4) now formulated for the functional $J$.

Remark 2. If we assume that $\Psi \geq 0$ on $\bar{\omega}$ and if we consider the discontinuous obstacle

$$
\hat{\Psi}:= \begin{cases}\Psi & \text { on } \omega \\ 0 & \text { on } \Omega-\omega,\end{cases}
$$

then it is easy to see that the solution $u$ of the problem

$$
I[\cdot, \Omega] \rightarrow \min \text { in } \mathbb{K}
$$

coincides with the unique solution $\hat{u}$ of

$$
\begin{aligned}
& I[\cdot, \Omega] \rightarrow \min _{\circ}^{\circ} \text { in } \hat{\mathbb{K}}, \\
& \hat{\mathbb{K}}:=\left\{w \in W_{p}^{1}(\Omega): w \geq \hat{\Psi} \quad \text { a.e. on } \Omega\right\} .
\end{aligned}
$$

\section{REFERENCES}

[1] R. A. Adams, Sobolev spaces, Academic Press, New York-San FranciscoLondon, 1975

[2] M. Bildhauer, M. Fuchs, G. Mingione, A priori gradient bounds and local $C^{1, \alpha}$-estimates for (double) obstacle problems under nonstandard growth conditions, Z. Anal. Anw. 20, no.4 (2001), 959-985. 
[3] E. Di Benedetto, $C^{1, \alpha}$ local regularity results of solutions of nonlinear degenerate elliptic equations, Nonlinear Analysis, TMA, 7 (1983), 827-850.

[4] H. J. Choe, J. L. Lewis, On the obstacle problem for quasilinear elliptic equations of $p$-Lalacian type, SIAM J. Math. Anal. 22, no 3, (1991), 623638.

[5] L. C. Evans, A new proof of local $C^{1, \alpha}$ regularity for solutions of certain degenerate P. D. E. s, J. Diff. Equ. 45 (1982), 356-373.

[6] S. Jagodziński, A. Olek, K. Szczepaniak, Lipschitz character of solutions to the inner obstacle problem, Irish Math. Soc. Bull. 61 (2008), 15-27.

[7] J. Jordanov, Solutions holderiennes d'inequations variationelles a constraines discontinuous, Serdica 8 (1982), 296-306.

[8] D. Kinderlehrer, G. Stampacchia, An introduction to variational inequalities and their applications, Academic Press, New York-London, 1980.

[9] G. Lieberman, Boundary regularity for solutions of degenerate elliptic equations, Nonlinear Analysis, TMA, 12 (1988), 1203-1219.

[10] G. Lieberman, Regularity of solutions to some degenerate double obstacle problems, Indiana Univ. Math. J. 40 (1991), 1009-1028.

[11] J. Manfredi, Regularity of the gradient for a class of nonlinear possibly degnerate equations, $\mathrm{PhD}$ thesis, Washington Univ. 1986.

[12] J. Manfredi, Regularity for minima of functionals with p-growth, J. Diff. Equ. 76 (1988), 203-212.

[13] J. Mu, W. P. Ziemer, Smooth regularity of solutions of double obstacle problems involving degenerate elliptic equations, Comm. P. D. E. 16 (485) (1991), 821-843.

[14] P. Tolksdorf, Regularity for a more general class of elliptic equations, J. Diff. Equ. 51 (1984), 126-150.

Martin Fuchs,

Department of Mathematics,

Saarland University,

P.O. Box 1511 50,

D-66041 Saarbrücken, Germany

fuchs@math.uni-sb.de

Received on 21 April 2009. 